Article

\title{
On a Length Problem for Univalent Functions
}

\author{
Mamoru Nunokawa ${ }^{1}$, Janusz Sokó ${ }^{2}{ }^{2}$ and Nak Eun Cho ${ }^{3, *}$ \\ 1 Department of Mathematics, Gunma University, Hoshikuki-cho 798-8, Chuou-Ward, Chiba 260-0808, Japan; \\ mamoru_nuno@doctor.nifty.jp \\ 2 Faculty of Mathematics and Natural Sciences, University of Rzeszów, 1 Prof. St. Pigoń Street, \\ 35-310 Rzeszów, Poland; jsokol@prz.edu.pl or jsokol@ur.edu.pl \\ 3 Department of Applied Mathematics, College of Natural Sciences, Pukyong National University, \\ Pusan 608-737, Korea \\ * Correspondence: necho@pknu.ac.kr
}

Received: 11 October 2018; Accepted: 16 November 2018; Published: 19 November 2018

\begin{abstract}
Let $g$ be an analytic function with the normalization in the open unit disk. Let $L(r)$ be the length of $g(\{z:|z|=r\})$. In this paper we present a correspondence between $g$ and $L(r)$ for the case when $g$ is not necessary univalent. Furthermore, some other results related to the length of analytic functions are also discussed.
\end{abstract}

Keywords: analytic functions; starlike functions; univalent functions; length problems

MSC: 30C45; 30C80

\section{Introduction}

Let $\mathcal{A}$ be the family of functions of the form

$$
g(z)=z+\sum_{n=2}^{\infty} a_{n} z^{n}
$$

which are analytic in the open unit disk $\mathbb{D}=\{z \in \mathbb{C}:|z|<1\}$. Let $\mathcal{S}$ denote the subfamily of $\mathcal{A}$ consisting of all univalent functions in $\mathbb{D}$.

Let $C(r)$ denote the image curve of the $|z|=r<1$ under the function $g \in \mathcal{A}$ which bound the area $A(r)$. Furthermore, let $L(r)$ be the length of $C(r)$ and $M(r)=\max _{|z|=r<1}|g(z)|$.

If $g \in \mathcal{A}$ satisfies

$$
\mathfrak{R e}\left\{\frac{z g^{\prime}(z)}{g(z)}\right\}>0, z \in \mathbb{D},
$$

then $g$ is said to be starlike with respect to the origin in $\mathbb{D}$ and we write $g \in \mathcal{S}^{*}$. It is known (for details, see $[1,2])$ that $\mathcal{S}^{*} \subset \mathcal{S}$.

The aim of the present paper is to prove, using a modified methodology, that in the following implication

$$
g \in \mathcal{S}^{*} \Rightarrow L(r)=\mathcal{O}\left(M(r) \log \frac{1}{1-r}\right) \quad \text { as } \quad r \rightarrow 1,
$$

where $\mathcal{O}$ denotes the Landau's symbol, the assumption that $g$ is starlike univalent can be changed by a weaker one. Result (2) was proved by Keogh [3]. Moreover, some other length problems for analytic functions are investigated. Several interesting developments related to length problems for univalent functions were considered in [4-15]. 


\section{Main Results}

Theorem 1. Let $g$ be of the form (1) and suppose that

$$
\left|\frac{z g^{\prime}(z)}{g(z)}\right| \leq\left|\frac{1+z}{1-z}\right|, \quad z \in \mathbb{D} \text {. }
$$

Then

$$
L(r)=\mathcal{O}\left(M(r) \log \frac{1}{1-r}\right) \quad \text { as } \quad r \rightarrow 1,
$$

where

$$
M(r)=\max _{|z|=r<1}|g(z)|
$$

and $\mathcal{O}$ means Landau's symbol.

Proof. Let $z=r e^{i v}$. We have $g \neq 0$ in $\mathbb{D} \backslash\{0\}$. In fact, if $g=0$ in $\mathbb{D}$, it contradicts hypothesis (3). Applying [3] (Theorem 1) and the hypothesis of Theorem 1, we have

$$
\begin{aligned}
L(r) & =\int_{0}^{2 \pi}\left|z g^{\prime}(z)\right| \mathrm{d} v=\int_{0}^{2 \pi}\left|\frac{z g^{\prime}(z)}{g(z)}\right||g(z)| \mathrm{d} v \\
& \leq M(r) \int_{0}^{2 \pi}\left|\frac{z g^{\prime}(z)}{g(z)}\right| \mathrm{d} v \leq M(r) \int_{0}^{2 \pi}\left|\frac{1+r e^{i v}}{1-r e^{i v}}\right| \mathrm{d} v \\
& \leq M(r)\left(2 \pi+4 \log \frac{1+r}{1-r}\right) \quad \text { as } \quad r \rightarrow 1 .
\end{aligned}
$$

Remark 1. If $g$ satisfies the condition of Theorem 1 , then $g$ is not necessary univalent in $\mathbb{D}$. It is well known that if $g \in \mathcal{S}$, then it follows that

$$
\frac{1-|z|}{1+|z|} \leq\left|\frac{z g^{\prime}(z)}{g(z)}\right| \leq \frac{1+|z|}{1-|z|}, \quad z \in \mathbb{D}
$$

(for details, see [1] (Vol. 1, p. 69)).

If $g \in \mathcal{A}$ satisfies

$$
\mathfrak{R e}\left\{\frac{z g^{\prime}(z)}{g^{1-\gamma}(z) h^{\gamma}(z)}\right\}>0, z \in \mathbb{D}
$$

for some $h \in \mathcal{S}^{*}$ and some $\gamma \in(0, \infty)$, then $g$ is said to be a Bazilevic function of type $\gamma$ [13]. The class of Bazilevic functions of type $\gamma$ is denoted by $g \in \mathcal{B}(\gamma)$. We note that Theorem 1 improves the implication (2) by Keogh [3] and it is also related to Theorem 3 given by Thomas [13].

We will need the following Tsuji's result.

Lemma 1 ([16] (p. 226)). (Theorem 3) If $0 \leq r<R$ and $z=e^{i v}$, then

$$
\frac{R-r}{R+r} \leq \mathfrak{R e}\left\{\frac{R e^{i \phi}+z}{R e^{i \phi}-z}\right\}=\frac{R^{2}-r^{2}}{R^{2}-2 R r \cos (\phi-v)+r^{2}} \leq \frac{R+r}{R-r}
$$

Moreover,

$$
\frac{1}{2 \pi} \int_{0}^{2 \pi} \frac{R^{2}-r^{2}}{R^{2}-2 R r \cos (\phi-v)+r^{2}} \mathrm{~d} v=1
$$


Theorem 2. Let $g$ be of the form (1) and suppose that

$$
\left|\frac{z g^{\prime}(z)}{g(z)}\right| \leq\left|\frac{1+z}{1-z}\right|, \quad z \in \mathbb{D}
$$

and

$$
M(r, \beta)=\max _{|z|=r<1}|g(z)| \leq\left|\frac{1+z}{1-z}\right|^{\beta},
$$

where $1<\beta$. Then

$$
L(r)=\mathcal{O}\left(\frac{1}{(1-r)^{\beta}}\right) \quad \text { as } \quad r \rightarrow 1,
$$

where $\mathcal{O}$ means Landau's symbol.

Proof. From the hypotheses (6) and (7), it follows that

$$
\begin{aligned}
L(r) & =\int_{0}^{2 \pi}\left|z g^{\prime}(z)\right| \mathrm{d} v=\int_{0}^{2 \pi}\left|\frac{z g^{\prime}(z)}{g(z)}\right||g(z)| \mathrm{d} v \\
& \leq \int_{0}^{2 \pi}\left|\frac{1+z}{1-z}\right|\left|\frac{1+z}{1-z}\right|^{\beta} \mathrm{d} v \leq 2^{1+\beta} \int_{0}^{2 \pi} \frac{1}{|1-z|^{1+\beta}} \mathrm{d} v \\
& =\frac{2^{1+\beta}}{(1-r)^{\beta-1}} \int_{0}^{2 \pi} \frac{1}{1-2 r \cos v+r^{2}} \mathrm{~d} v .
\end{aligned}
$$

From (5), we have

$$
\int_{0}^{2 \pi} \frac{1}{1-2 r \cos v+r^{2}} \mathrm{~d} v=\frac{2 \pi}{1-r^{2}}
$$

Hence, we obtain

$$
\begin{aligned}
L(r) & \leq \frac{2^{1+\beta}}{(1-r)^{\beta-1}} \frac{2 \pi}{1-r^{2}} \\
& =\mathcal{O}\left(\frac{1}{(1-r)^{\beta}}\right) \text { as } r \rightarrow 1 .
\end{aligned}
$$

Therefore, we complete the proof of Theorem 2.

Let us recall the following Fejér-Riesz's result.

Lemma 2 ([16]). Let $h$ be analytic in $\mathbb{D}$ and continuous on $\overline{\mathbb{D}}$. Then

$$
\int_{-1}^{1}|h(z)|^{p}|\mathrm{~d} z| \leq \frac{1}{2} \int_{|z|=1}|h(z)|^{p}|\mathrm{~d} z|
$$

where $p>0$.

Theorem 3. Let $g$ be of the form (1) and suppose that

$$
\frac{1-|z|}{1+|z|} \leq\left|\frac{z g^{\prime}(z)}{g(z)}\right| \leq \frac{1+|z|}{1-|z|}, \quad z \in \mathbb{D}
$$

Then

$$
\mathcal{O}\left(m(r) \log \frac{1}{1-r}\right) \leq L(r) \leq \mathcal{O}\left(\frac{M(r)}{1-r}\right) \quad \text { as } \quad r \rightarrow 1
$$

where

$$
m(r)=\min _{|z|=r<1}|g(z)|, \quad M(r)=\max _{|z|=r<1}|g(z)|
$$


and $\mathcal{O}$ means Landau's symbol.

Proof. From the assumption, we have

$$
\begin{aligned}
L(r) & =\int_{0}^{2 \pi}\left|z g^{\prime}(z)\right| \mathrm{d} v=\int_{0}^{2 \pi}\left|\frac{z g^{\prime}(z)}{g(z)}\right||g(z)| \mathrm{d} v \\
& \geq m(r) \int_{0}^{2 \pi}\left|\frac{z g^{\prime}(z)}{g(z)}\right| \mathrm{d} v
\end{aligned}
$$

because $g(z) \neq 0$ in $\mathbb{D} \backslash\{0\}$. In fact, if $g(z)=0$ in $\mathbb{D}$, it contradicts hypothesis (8). Applying Fejér-Riesz's Lemma 2, we have

$$
\begin{aligned}
L(r) & \geq m(r) \int_{0}^{2 \pi}\left|\frac{z g^{\prime}(z)}{g(z)}\right| \mathrm{d} v \geq 2 m(r) \int_{-r}^{r} \frac{1-\rho}{1+\rho} \mathrm{d} \rho \\
& \geq 2 m(r) \log \frac{1+r}{1-r}-2 r \\
& =\mathcal{O}\left(m(r) \log \frac{1}{(1-r)}\right) \quad \text { as } \quad r \rightarrow 1 .
\end{aligned}
$$

While, we obtain

$$
\begin{aligned}
L(r) & =\int_{0}^{2 \pi}\left|z g^{\prime}(z)\right| \mathrm{d} v=\int_{0}^{2 \pi}\left|\frac{z g^{\prime}(z)}{g(z)}\right||g(z)| \mathrm{d} v \\
& =M(r) \int_{0}^{2 \pi} \frac{1+|z|}{1-|z|} \mathrm{d} v=2 \pi M(r) \frac{1+r}{1-r} \\
& =\mathcal{O}\left(\frac{M(r)}{1-r}\right) \quad \text { as } \quad r \rightarrow 1 .
\end{aligned}
$$

Therefore, we complete the proof of Theorem 3.

From Theorem 3, we have the following result.

Corollary 1. Let $g$ be of the form (1) and suppose that $g$ is univalent in $\mathbb{D}$. Then we have

$$
\mathcal{O}\left(m(r) \log \frac{1}{1-r}\right) \leq L(r) \leq \mathcal{O}\left(\frac{M(r)}{1-r}\right) \quad \text { as } \quad r \rightarrow 1,
$$

where $m(r)$ and $M(r)$ are given by (9), respectively.

Proof. From the hypothesis, we have

$$
\frac{1-|z|}{1+z \mid} \leq\left|\frac{z g^{\prime}(z)}{g(z)}\right| \leq \frac{1+|z|}{1-|z|}, \quad z \in \mathbb{D},
$$

which completes the proof.

Lemma 3 ([17] (p. 280) and [18] (p. 491)).

$$
\int_{0}^{2 \pi} \frac{\mathrm{d} v}{\left|1-r e^{i v}\right|^{\beta}}= \begin{cases}\mathcal{O}\left((1-r)^{1-\beta}\right) & \text { for the case } 1<\beta, \\ \mathcal{O}\left(\log \frac{1}{1-r}\right) & \text { for the case } \beta=1 \\ \mathcal{O}(1) & \text { for the case } 0 \leq \beta<1\end{cases}
$$


where $0<r<1,0 \leq v \leq 2 \pi, 0 \leq \beta$ and $\mathcal{O}$ means Landau's symbol.

Theorem 4. Let $g$ be of the form (1) and suppose that

$$
\left|\frac{z g^{\prime}(z)}{g(z)}\right| \leq \frac{1}{1-|z|}, \quad z \in \mathbb{D}
$$

and

$$
|g(z)| \leq \frac{1}{|1-z|^{\beta}}, \quad z \in \mathbb{D}
$$

Then

$$
L(r) \leq \begin{cases}\mathcal{O}\left((1-r)^{-3 / 2}\right) & \text { for } 1<\beta \leq 3 / 2, \\ \mathcal{O}\left((1-r)^{-3 / 2} \log \frac{1}{1-r}\right) & \text { for the case } \beta=3 / 2, \\ \mathcal{O}\left((1-r)^{-\beta}\right) & \text { for the case } 3 / 2<\beta,\end{cases}
$$

where $0<|z|=r<1$ and $\mathcal{O}$ means Landau's symbol.

Proof. From the hypothesis (10), it follows that $g(z) \neq 0$ in $\mathbb{D} \backslash\{0\}$. Then we have

$$
\begin{aligned}
L(r) & =\int_{0}^{2 \pi}\left|r e^{i v} g^{\prime}\left(r e^{i v}\right)\right| \mathrm{d} v=\int_{0}^{2 \pi}\left|\frac{z g^{\prime}(z)}{g(z)}\right||g(z)| \mathrm{d} v \\
& <\int_{0}^{2 \pi}\left(\frac{1}{1-|z|}\right)\left(\frac{1}{|1-z|^{\beta}}\right) \mathrm{d} v \\
& =\int_{0}^{2 \pi}\left(\frac{1}{|1-z|}\right)\left(\frac{1}{|1-z|^{\beta-1}}\right)\left(\frac{1}{1-|z|}\right) \mathrm{d} v \\
& \leq\left(\int_{0}^{2 \pi} \frac{1}{|1-z|^{2}} \mathrm{~d} v\right)^{1 / 2}\left(\int_{0}^{2 \pi}\left(\frac{1}{|1-z|^{2 \beta-2}}\right) \frac{1}{(1-|z|)^{2}} \mathrm{~d} v\right)^{1 / 2} .
\end{aligned}
$$

Applying Hayman's Lemma 3, we have

$$
\begin{aligned}
L(r) & \leq\left(\frac{1}{1-r^{2}}\right)^{1 / 2}\left(\frac{1}{1-r}\right) \mathcal{O}(1) \\
& =\mathcal{O}\left(\frac{1}{(1-r)^{3 / 2}}\right) \quad \text { as } \quad r \rightarrow 1
\end{aligned}
$$

for the case $1<\beta<3 / 2$,

$$
\begin{aligned}
L(r) & \leq\left(\frac{1}{1-r^{2}}\right)^{1 / 2}\left(\frac{1}{1-r}\right) \mathcal{O}\left(\log \frac{1}{1-r}\right) \\
& =\mathcal{O}\left(\frac{1}{(1-r)^{3 / 2}} \log \frac{1}{1-r}\right) \quad \text { as } \quad r \rightarrow 1
\end{aligned}
$$

for the case $\beta=3 / 2$ and

$$
L(r)=\left(\frac{1}{1-r^{2}}\right)^{1 / 2}\left(\frac{1}{1-r}\right)\left(\frac{1}{1-r}\right)^{(2 \beta-3) / 2} \text { as } \quad r \rightarrow 1
$$

for the case $3 / 2<\beta$.

Lemma 4 ([16] (p. 227)). If $g(z)=u(z)+i v(z)$ is analytic in $|z| \leq R$, then

$$
g(z)=\frac{1}{2 \pi} \int_{0}^{2 \pi} u\left(R e^{i \phi}\right) \frac{R e^{i \phi}+z}{R e^{i \phi}-z} \mathrm{~d} \phi+i v(0) .
$$


Moreover, if $|z|<R$ and $v(0)=0$, then

$$
|g(z)|=\frac{1}{2 \pi} \int_{0}^{2 \pi}\left|u\left(R e^{i \phi}\right)\right| \frac{R e^{i \phi}+z}{R e^{i \phi}-z} \mid \mathrm{d} \phi .
$$

Theorem 5. Let $g$ be of the form (1). Then

$$
M(r)=\mathcal{O}\left(A(r) \log \frac{1}{1-r}\right) \text { as } r \rightarrow 1,
$$

where $0<|z|=r<1$ and $\mathcal{O}$ means Landau's symbol.

Proof. It follows that

$$
M(r)=\max _{|z|=r<1}\left|\int_{0}^{z} g^{\prime}(s) \mathrm{d} s\right|=\max _{|z|=r<1}\left|\int_{0}^{r} g^{\prime}\left(\rho e^{i v}\right) \mathrm{d} \rho\right| .
$$

Applying (12), we have

$$
\begin{aligned}
M(r) & =\max _{|z|=r<1}\left|\frac{1}{2 \pi} \int_{0}^{r} \int_{0}^{2 \pi} \mathfrak{R e} g^{\prime}\left(t e^{i v}\right) \frac{t e^{i \phi}+\rho e^{i v}}{t e^{i \phi}-\rho e^{i v}} \mathrm{~d} \phi \mathrm{d} \rho\right| \\
& \leq \max _{|z|=r<1} \frac{1}{2 \pi} \int_{0}^{r} \int_{0}^{2 \pi}\left|g^{\prime}\left(t e^{i v}\right)\right|\left|\frac{t e^{i \phi}+\rho e^{i v}}{t e^{i \phi}-\rho e^{i v}}\right| \mathrm{d} \phi \mathrm{d} \rho
\end{aligned}
$$

where $0 \leq \rho \leq r<t<1$. Then, applying Schwarz's lemma, we have

$$
\begin{aligned}
M(r) & \leq \max _{|z|=r<1}\left(\frac{1}{2 \pi} \int_{0}^{r} \int_{0}^{2 \pi}\left|g^{\prime}\left(t e^{i v}\right)\right|^{2} \mathrm{~d} \phi \mathrm{d} \rho\right)^{1 / 2}\left(\int_{0}^{r} \int_{0}^{2 \pi}\left|\frac{t e^{i \phi}+\rho e^{i v}}{t e^{i \phi}-\rho e^{i v}}\right|^{2} \mathrm{~d} \phi \mathrm{d} \rho\right)^{1 / 2} \\
& \leq \max _{|z|=r<1}\left(I_{1}\right)^{1 / 2}\left(I_{2}\right)^{1 / 2}, \text { say. }
\end{aligned}
$$

Putting $0<r_{1}<r$ and $t=\sqrt{\left(1+\rho^{2}\right) / 2}$, we have

$$
\rho \mathrm{d} \rho=2 \sqrt{\frac{1+\rho^{2}}{2}} \mathrm{~d} t<2 \mathrm{~d} t .
$$

Then we have

$$
\begin{aligned}
I_{1} & =\frac{1}{2 \pi} \int_{0}^{r_{1}} \int_{0}^{2 \pi}\left|g^{\prime}\left(t e^{i \phi}\right)\right|^{2} \mathrm{~d} \phi \mathrm{d} \rho+\frac{1}{2 \pi r_{1}^{2}} \int_{\sqrt{\left(1+r_{1}^{2}\right) / 2}}^{\sqrt{\left(1+r^{2}\right) / 2}} \int_{0}^{2 \pi} t\left|g^{\prime}\left(t e^{i \phi}\right)\right|^{2} \mathrm{~d} \phi \mathrm{d} t \\
& \leq C+\frac{1}{2 \pi r_{1}^{2}} A\left(\sqrt{\frac{1+r^{2}}{2}}\right) \\
& =C+\frac{1}{2 \pi r_{1}^{2}} A\left(\sqrt{\frac{1+r^{2}}{2 r^{2}}} r\right) \\
& =\mathcal{O}(A(r)) \text { as } r \rightarrow 1,
\end{aligned}
$$


where $C$ is a bounded positive constant. On the other hand, putting $t \rightarrow 1^{-}$, we have

$$
\begin{aligned}
I_{2} & =\int_{0}^{r} \int_{0}^{2 \pi}\left|\frac{t e^{i \phi}+\rho e^{i v}}{t e^{i \phi}-\rho e^{i v}}\right|^{2} \mathrm{~d} \phi \mathrm{d} \rho \\
& \leq \int_{0}^{r} \int_{0}^{2 \pi} \frac{4}{\left|t e^{i \phi}-\rho e^{i v}\right|^{2}} \mathrm{~d} \phi \mathrm{d} \rho \\
& =\int_{0}^{r} \int_{0}^{2 \pi} \frac{4}{t^{2}-2 \rho t \cos (\phi-v)+\rho^{2}} \mathrm{~d} \phi \mathrm{d} \rho .
\end{aligned}
$$

Using (5), we have

$$
\begin{aligned}
I_{2} & \leq 8 \pi \int_{0}^{r} \frac{1}{t^{2}-\rho^{2}} \mathrm{~d} \rho \\
& =\frac{4 \pi}{t} \int_{0}^{r}\left(\frac{1}{t+\rho}+\frac{1}{t-\rho}\right) \mathrm{d} \rho \\
& =\frac{4 \pi}{t} \log \frac{t+r}{t-r} \rightarrow \mathcal{O}\left(\log \frac{1}{1-r}\right) \text { as } r \rightarrow 1 .
\end{aligned}
$$

Therefore we complete the proof of (13).

Remark 2. In Theorem 5, we do not suppose that $g$ is univalent in $|z|<1$ and therefore, it improves the result by Pommerenke [2].

Author Contributions: All authors contributed equally.

Funding: The authors would like to express their gratitude to the referees for many valuable suggestions regarding the previous version of this paper. This research was supported by the Basic Science Research Program through the National Research Foundation of Korea (NRF) funded by the Ministry of Education, Science and Technology (No. 2016R1D1A1A09916450).

Conflicts of Interest: The authors declare no conflict of interest.

\section{References}

1. Goodman, A.W. Univalent Functions; Mariner Publishing Co.: Tampa, FL, USA, 1983.

2. Pommerenke, C. Über nahezu konvexe analytische Functionen. Arch. Math. 1965, 16, 344-347. [CrossRef]

3. Keogh, F.R. Some theorems on conformal mapping of bounded star-shaped domain. Proc. Lond. Math. Soc. 1959, 9, 481-491. [CrossRef]

4. Guariglia, E. Fractional Derivative of the Riemann zeta function. In Fractional Dynamics; Cattani, C., Srivastava, H.M., Yang, X.J., Eds.; De Gruyter: Berlin, Germany, 2015; Volume 21, pp. 357-368.

5. Guariglia, E.; Silvestrov, S. A functional equation for the Riemann zeta fractional derivative. AIP Conf. Proc. 2017, 1798, 020063.

6. Lian, H.H.; Ge, W. Calculus of variations for a boundary value problem of differential system on the half line. Math. Comput. Appl. 2009, 58, 58-64. [CrossRef]

7. Nunokawa, M. On Bazilevič and convex functions. Trans. Am. Math. Soc. 1969, 143, 337-341.

8. Nunokawa, M. A note on convex and Bazilevič functions. Proc. Am. Math. Soc. 1970, 24, 332-335.

9. Nunokawa, M.; Owa, S.; Hayami, T.; Kuroki, K. Some properties of univalent functions. Int. J. Pure Appl. Math. 2009, 52, 603-609.

10. Nunokawa, M.; Sokół, J. On some lenght problems for analytic functions. Osaka J. Math. 2014, 51, 695-707.

11. Nunokawa, M.; Sokół, J. On some lenght problems for univalent functions. Math. Meth. Appl. Sci. 2016, 39, 1662-1666. [CrossRef]

12. Nunokawa, M.; Sokót, J. On some lenght problems for close-to-convex functions. Stud. Sci. Math. Hung. 2018, in press.

13. Thomas, D.K. On Bazilevič functions. Trans. Am. Math. Soc. 1968, 132, 353-361. 
14. Thomas, D.K. On starlike and close-to-convex univalent functions. J. Lond. Math. Soc. 1967, 42, 427-435. [CrossRef]

15. Thomas, D.K. A note on starlike functions. J. Lond. Math. Soc. 1968, 43, 703-706. [CrossRef]

16. Tsuji, M. Complex Functions Theory; Maki Book Company: Tokyo, Japan, 1968. (In Japanese)

17. Hayman, W.F. The asymptotic behaviour of $p$-valent functions. Proc. Lond. Math. Soc. 1955, 3, $257-284$. [CrossRef]

18. Eenigenburg, P. On the radius of curvature for convex analytic functions. Can. J. Math. 1970, 22, 486-491. [CrossRef]

(C) 2018 by the authors. Licensee MDPI, Basel, Switzerland. This article is an open access article distributed under the terms and conditions of the Creative Commons Attribution (CC BY) license (http:/ / creativecommons.org/licenses/by/4.0/). 